\title{
Electrochemical Waves on Patterned Surfaces: Propagation through Narrow Gaps and Channels
}

\author{
Konstantin Agladze, ${ }^{\dagger}$ Stephanie Thouvenel-Romans, and Oliver Steinbock* \\ Department of Chemistry, The Florida State University, Tallahassee, Florida 32306-4390
}

Received: April 9, 2001; In Final Form: May 29, 2001

\begin{abstract}
The dissolution and corrosion of iron and certain types of steel can involve the propagation of electrochemical waves. Low-carbon steel plates covered by nitric acid solutions self-organize pseudo-two-dimensional wave patterns which share many features with reaction-diffusion waves in excitable systems. Here, we investigate the dynamics of these electrodissolution waves in the presence of geometrical constraints. A simple procedure for the preparation of insulating, millimeter-scale obstacles is presented, which allows the controlled, local protection of the metal surface. This method is used in the measurement of the material consumption rate per wave $\left[(850 \pm 50) \mathrm{nm} /\right.$ wave or $6-7 \mathrm{~g} /\left(\mathrm{m}^{2}\right.$ wave $\left.)\right]$ as well as for the study of front propagation through narrow gaps and long channels. In channels, the wave velocity decreases with decreasing width from 10 to $4 \mathrm{~mm} / \mathrm{s}$. Propagation failures occur within the channel if its width is smaller than $0.2-0.4 \mathrm{~mm}$. These results indicate that the obstacles have sink-like boundaries. For the estimation of the critical nucleation size in this system, we prepared short and narrow gaps that induce wave blockage for openings smaller than $0.65 \pm 0.1 \mathrm{~mm}$.
\end{abstract}

\section{Introduction}

Spatiotemporal patterns are found in a variety of nonequilibrium systems and range from stationary Turing structures to chemical turbulence. ${ }^{1-3}$ An essential feature for pattern formation in these systems is that the underlying reaction mechanism involves nonlinearities such as autocatalysis or surface-phase transitions. The second, important feature is that the local reaction dynamics must be spatially coupled by transport processes. Two widely studied chemical systems that fulfill these criteria are the Belousov-Zhabotinsky reaction ${ }^{4,5}$ and the $\mathrm{CO}$ oxidation on platinum. ${ }^{6,7}$ These reaction-diffusion systems as well as many other chemical and biological media show dynamically similar phenomena such as propagating fronts, target patterns, and rotating spiral waves.

Pattern formation also occurs in electrochemical systems where it has attracted considerable interest. ${ }^{8-10}$ Electrochemistry is an ideal playground for the study of dynamic instabilities because here most experiments are carried out under conditions far from thermodynamic equilibrium. It is therefore not surprising that the majority of known bistable, oscillating, and chaotic reactions is found in electrochemistry. ${ }^{8}$ In addition, spatial coupling is intrinsic to most processes that occur on the surface of electrodes because local changes in the double-layer potential induce migration currents that can affect significantly the dynamics at other locations. The range and the strength of this spatial coupling are determined by parameters such as the cell geometry, the external resistance, and the conductivity of the electrolyte. ${ }^{10}$ In many standard electrochemical applications, the setup creates strong, long-range coupling through the electrolyte, which hampers the formation of pronounced spatial patterns. For the case of weak, long-range coupling, however, complex spatiotemporal oscillations ${ }^{11-14}$ as well as accelerating ${ }^{15}$ and "remote-triggered" fronts ${ }^{16}$ have been observed. Short-range or

* To whom correspondence should be addressed.

On leave from Institute of Theoretical and Experimental Biophysics, Pushchino, Moscow Region 142292, Russia. local coupling can be achieved by establishing an equipotential surface (e.g., the counter electrode) or similar boundary conditions in close distance to the working electrode. Consequently, changes in a particular point affect only its immediate neighborhood, which is formally similar to diffusion-induced coupling. Under these conditions, traveling fronts and other structures can form on the electrode surface that are closely related to patterns known from reaction-diffusion media.

The classic examples of wave propagation in electrochemical systems are found for the electrodissolution of certain metals and alloys such as nickel, cobalt, iron, and steel. ${ }^{18-21}$ The surface of these metals can be passivated by a self-organized oxide film which prevents or minimizes dissolution. ${ }^{22,23}$ A local damage of this protective film, however, forms an anodic region which can nucleate a propagating front. This type of electrochemical wave was first discovered for the iron-nitric acid system where dissolution fronts travel along metal wires with speeds of up to $4 \mathrm{~m} / \mathrm{s} .{ }^{21}$ Each dissolution front is typically followed by a trailing repassivation zone in which the excitable (passive) state of the surface is recovered. It should be noted that the repassivation front can move faster than the leading activation front. ${ }^{24}$ The formation of electrochemical waves is not limited to thin metal wires but has also been observed on planar electrode surfaces. For example, Otterstedt et al. studied the dissolution of cobalt ring and disk electrodes and detected fronts that rotated around a central reference electrode. ${ }^{25,26}$ More recently, rotating spiral waves have been observed under open-circuit conditions on nitric acid covered, low-carbon steel plates. ${ }^{27}$ These vortices are similar to spiral waves in reaction-diffusion systems. Therefore, the iron-nitric acid reaction seems to be a good model for the detailed investigation of electrochemical wave propagation under short-range coupling. However, it should be noted that the employed open-circuit conditions limit the controllability of the system.

In this paper, we investigate the influence of geometrical constraints on the traveling waves in the iron-nitric acid system. The broader motivation for applying such constraints to a self- 
organizing medium stems from the desire to optimize the catalytic performance of a system or to stabilize its dynamic behavior in terms of controlled pattern formation. This general approach has therefore caught considerable interest in the field of heterogeneous-catalysis research. ${ }^{6,28}$ In addition, studies of the Belousov-Zhabotinsky reaction demonstrate that obstaclepatterned excitable media can induce anisotropic front propagation, ${ }^{29}$ nucleate spiral waves, ${ }^{29,30}$ or mimic certain features of heterogeneous cell cultures. ${ }^{31}$ Moreover, experiments with waves exiting from thin channels into large systems have proven to be a valuable tool for the analysis of reaction fronts. ${ }^{32-34}$ These experiments yield the critical (i.e., minimal) radius of a superthreshold perturbation required for successful wave initiation. The existence of such a critical radius is well established for reaction-diffusion systems. ${ }^{35,36}$ However, neither experimental nor theoretical studies have been dedicated to the critical size of perturbations in electrochemical systems, and also, the systematic investigation of patterned electrodes is still in its infancy. ${ }^{37}$ The main purpose of this paper derives itself from this lack of experimental analyses and the prospect of obtaining useful insights into the dynamic stability of passivation films against localized perturbations.

\section{Experimental Section}

All experiments are carried out with low-carbon steel plates (McMaster-Carr -C1018: $\sim 0.15-0.20 \%$ C , $\sim 0.60-0.90 \%$ $\mathrm{Mn},<0.040 \% \mathrm{P},<0.050 \% \mathrm{~S})$ which are covered by a thin $(\sim 0.3$ $\mathrm{mm}$ ) layer of nitric acid (Fisher). Two different concentrations of nitric acid (11.2 and $11.9 \mathrm{M})$ are considered in this investigation. The dimensions of the steel plates are $5.1 \times 10.2$ $\times 0.3 \mathrm{~cm}$. No external electrodes are employed. As described in a recent paper, ${ }^{27}$ this open-circuit system can form propagating waves of dissolution that are readily detected by the unaided eye. This feature allows the use of optical techniques that yield data of high spatial resolution. Here, we used a monochrome CCD (charged coupled device) camera and digitized the video signals with a PC-based frame grabber board (Data Translation; $640 \times 480$ pixel resolution with $8 \mathrm{bit} / \mathrm{pixel}$ ) for further image processing. The complementing measurements of dissolution rates are carried out with a profilometer (Tencor, Alpha-Step 200).

At room temperature, the reaction of iron with nitric acid can be very violent. Fast waves of active dissolution appear spontaneously and render the system uncontrollable and dangerous to handle. Along with a rapid dissolution of the metal, a strong release of thermal energy exacerbates the problem. To slow the reaction, the temperature of the system is kept constant at $0{ }^{\circ} \mathrm{C}$ during all stages of the experiment. The detailed experimental procedure involves three different phases: (i) the prepassivation of the steel plates, (ii) the preparation of the obstacles, and last (iii) the main experiment.

Initial attempts showed that it is useful to passivate the steel surface before any obstacles are applied. This prepassivation is carried out in solutions of nitric acid in which the concentration of the acid equals the one in the subsequent experiment. The plates are immersed in the solution and left undisturbed to passivate for $10 \mathrm{~min}$. After this time, they are transferred to a distilled water cleaning dip. This step is necessary to avoid the spontaneous formation of waves during the sudden change in the environment. The plates are rinsed with acetone to remove any dissolved metal that remained on the surface and quickly dried to prevent corrosion.

After completion of this prepassivation procedure, thin, insulating obstacles are applied to the steel surface using pressure sensitive adhesive. We explored numerous approaches to create suitable obstacles that show sufficient acid resistance. On the basis of these experiments, we converged to a very simple technique in which masks of transparent, acid-resistant film are cut from common self-adhering plastic tape $(3 \mathrm{M})$ to form the desired obstacles. Two basic shapes of obstacles are devised to study the wave propagation in this system. Both of them divide the steel surface into two equally large domains with a narrow opening connecting them. The first type of obstacle creates a "channel" between the two domains. It is a long $(1.9 \mathrm{~cm})$ but narrow $(0.2-4.1 \mathrm{~mm})$ gap between the parallel edges of two rectangular obstacles. The second type of external constraint will be referred to as "gate" and consists of two triangular obstacles. Their slanted sides create a K-shaped structure with a narrow passage of very short length (less than $2 \mathrm{~mm})$ at the center. The steepest angle $\left(\sim 30^{\circ}\right)$ of the triangles points toward the center of the opening. The tips of the triangles are slightly truncated to ensure better adhesive properties of the mask. Using an optical microscope, the width of the opening $(0.2$ and $2.5 \mathrm{~mm})$ is measured before and after the experiment. In a few experiments, the mask became partially removed from the metal surface and the corresponding data had to be discarded. In most cases, however, the obstacles adhered well to the metal and the channel width remained constant throughout the experiment.

The actual wave experiments are conducted according to the following procedure. A prepassivated, obstacle-patterned plate is placed into the nitric acid solution. After the sample has reached thermal equilibrium, a first wave is initiated by touching the nitric-acid covered surface with a zinc ribbon. Notice that the thickness of the nitric acid layer is sufficient to wet the entire upper surface of the obstacle. We found that the first wave propagates slightly faster than the subsequent ones. For this reason, data from the first wave are excluded from the presented analyses, with the exception of the measurements of dissolution rates. The waves are initiated approximately $2 \mathrm{~cm}$ from the entrance of the obstacle, which is sufficient to obtain a nearly linear front at the gap. After each wave, the sample is again submerged into the nitric acid solution and left to recover for at least $2 \mathrm{~min}$. Each particular steel sample is typically used for five experimental runs.

\section{Results}

Figure 1 shows four consecutive snapshots of propagating waves on a nitric acid covered steel surface. In the experiment, the waves appear as brownish dark bands that travel over the grayish steel surface with speeds of approximately $1 \mathrm{~cm} / \mathrm{s}$. This particular example reveals two spiral waves with opposite sense of rotation. A large, square obstacle, in the upper right-hand section of the figures constraints the space accessible for propagating waves by electrically insulating the metal surface and by mechanically protecting it from dissolution. The counterclockwise rotating spiral on the left (most pronounced in Figure 1d) evolves without any signs of interference from the distant barrier. The tip of the second spiral wave, however, clearly follows the boundary of the insulating obstacle. Notice that the spiral tip extends all of the way to the boundary, which indicates that no significant amounts of inhibitory species are released from the plastic film into the system. The transparent character of the film allows us to further evaluate the state of the metal underneath the obstacle. These visual observations show no evidence for active corrosion that could conceivably emanate from the dissolution wave into the protected surface area. 

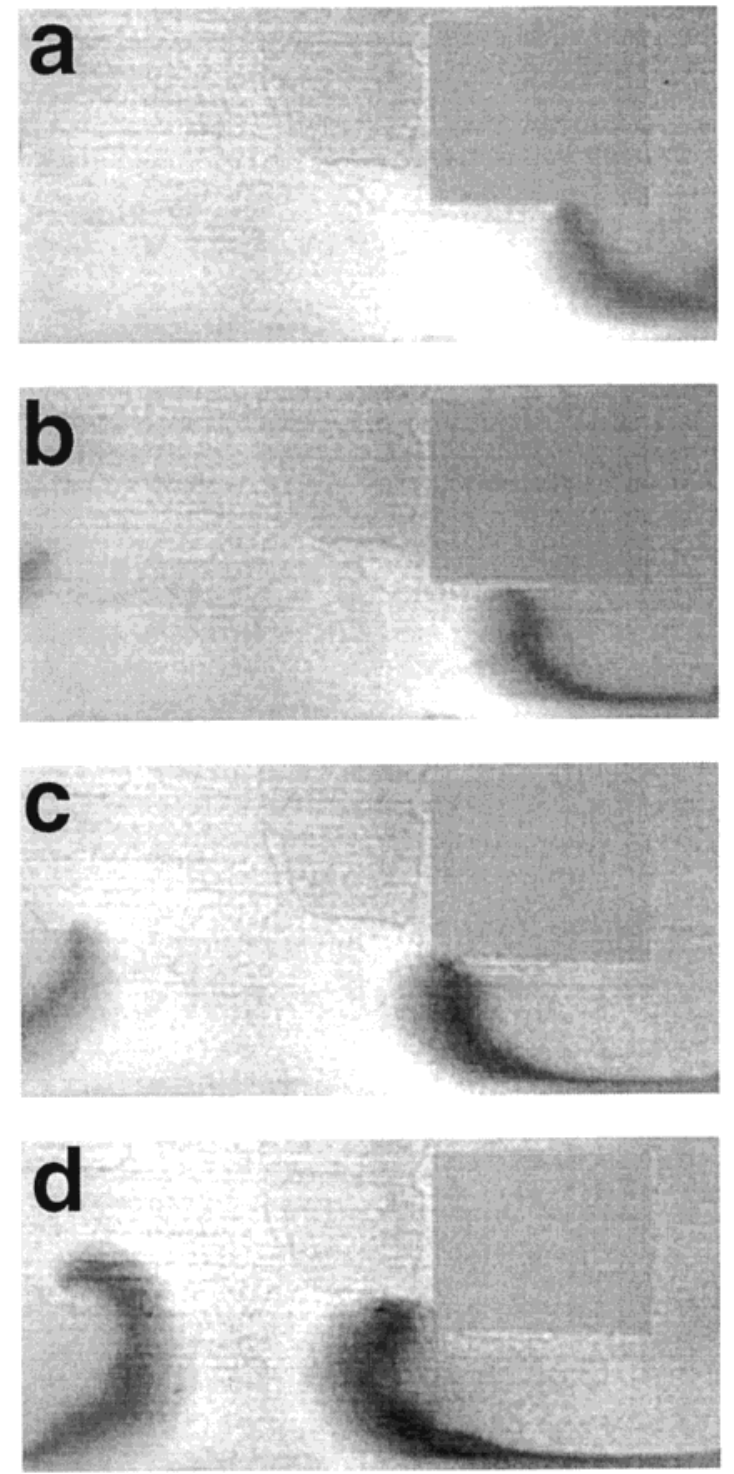

Figure 1. Wave propagation on the surface of nitric acid covered steel. The sequence shows two rotating spiral waves of dissolution. The spiral on the right-hand side travels along the boundary of a thin, rectangular obstacle. Image size, $6.1 \times 2.9 \mathrm{~cm}^{2}$; time between frames, $0.4 \mathrm{~s}$; [ $\left.\mathrm{HNO}_{3}\right]$ $=11.9 \mathrm{M}$.

To obtain further insights into the specific nature of the obstacle-wave interaction, we carried out systematic measurements of the front-mediated material consumption. In these experiments, 5-50 waves are sequentially initiated on steel surfaces that hold simple rectangular obstacles. After completion of the experiment, the plastic film is carefully removed from each plate. The resulting steel samples show striking patterns on their surfaces, which match the original geometry of the obstacle. We observed that the uncovered areas have a brighter somewhat polished character, whereas the light reflection from the protected areas is unchanged with respect to the original plates.

The surface topography of the samples is analyzed using a profilometer. Figure 2 shows typical height profiles across the boundary of the (removed) obstacles. The two plots differ in the number of waves, $N$, that were initiated on the corresponding samples ( $N=30$ and 50 in Figure 2 parts a and b, respectively). As expected, we observe a loss of material in the unprotected region $(x<1000 \mu \mathrm{m})$, which gives rise to a step in the surface height. None of the numerous samples used in this study showed any indications for dissolution underneath the obstacle. A
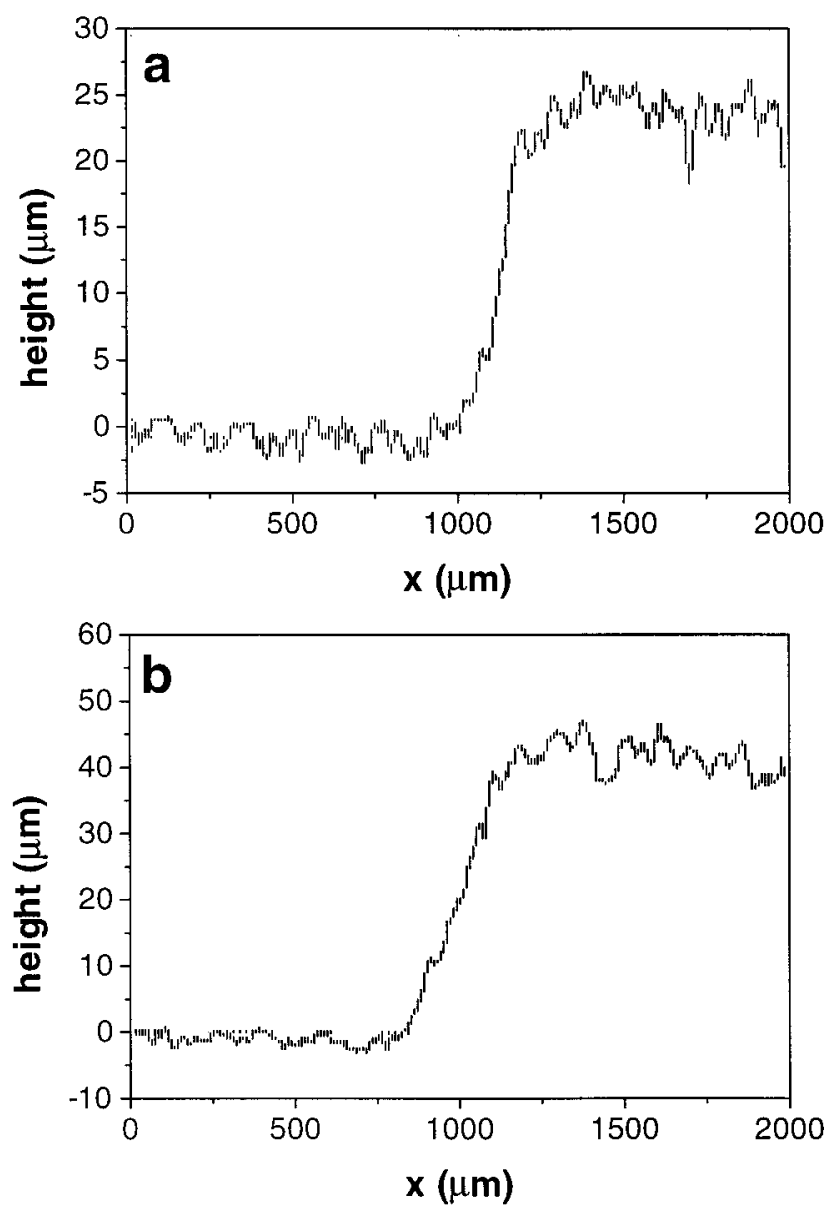

Figure 2. Height profiles across the boundary of insulating obstacles. The steps are created by collisions of numerous dissolution waves with the boundaries of the insulating obstacles. The obstacles were removed from the surface prior to the profilometric measurements. The total number of the waves are $N=30$ in (a) and $N=50$ in (b).

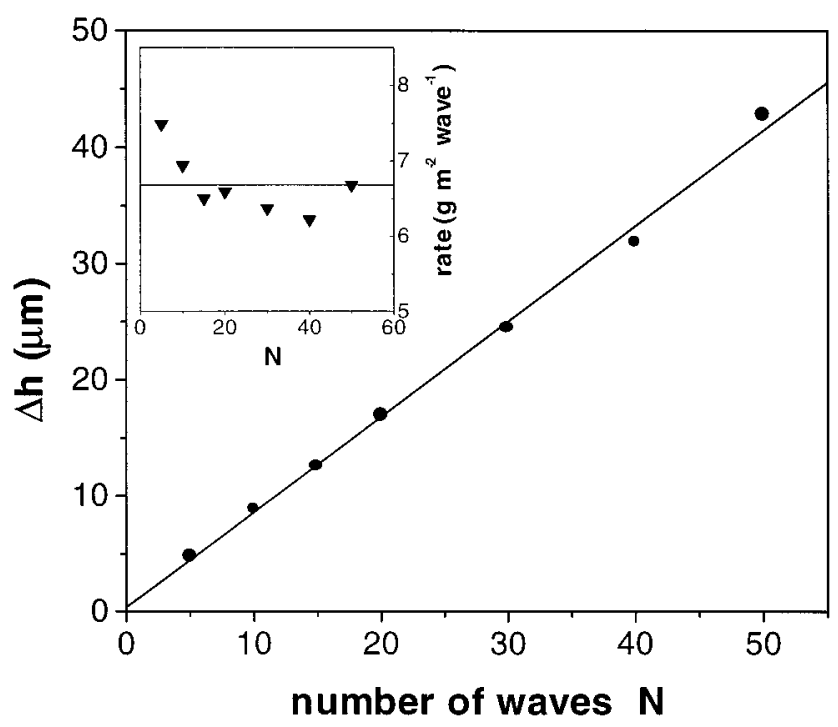

Figure 3. Height difference of the surface step $(\Delta h)$, which is proportional to the total numbers of electrodissolution waves $(N)$ creating the step at the boundary of an obstacle. The inset shows the corresponding consumption rates per wave and unit area. The data are obtained for $\left[\mathrm{HNO}_{3}\right]=11.2 \mathrm{M}$ and $T \approx 0{ }^{\circ} \mathrm{C}$.

comparison of the step heights in Figure 2 reveals that the height difference increases with the number of dissolution waves that annihilate at the obstacle. It should be noted that the step width (200 $\mu \mathrm{m}$ in Figure 2a) is significantly decreased for smaller 

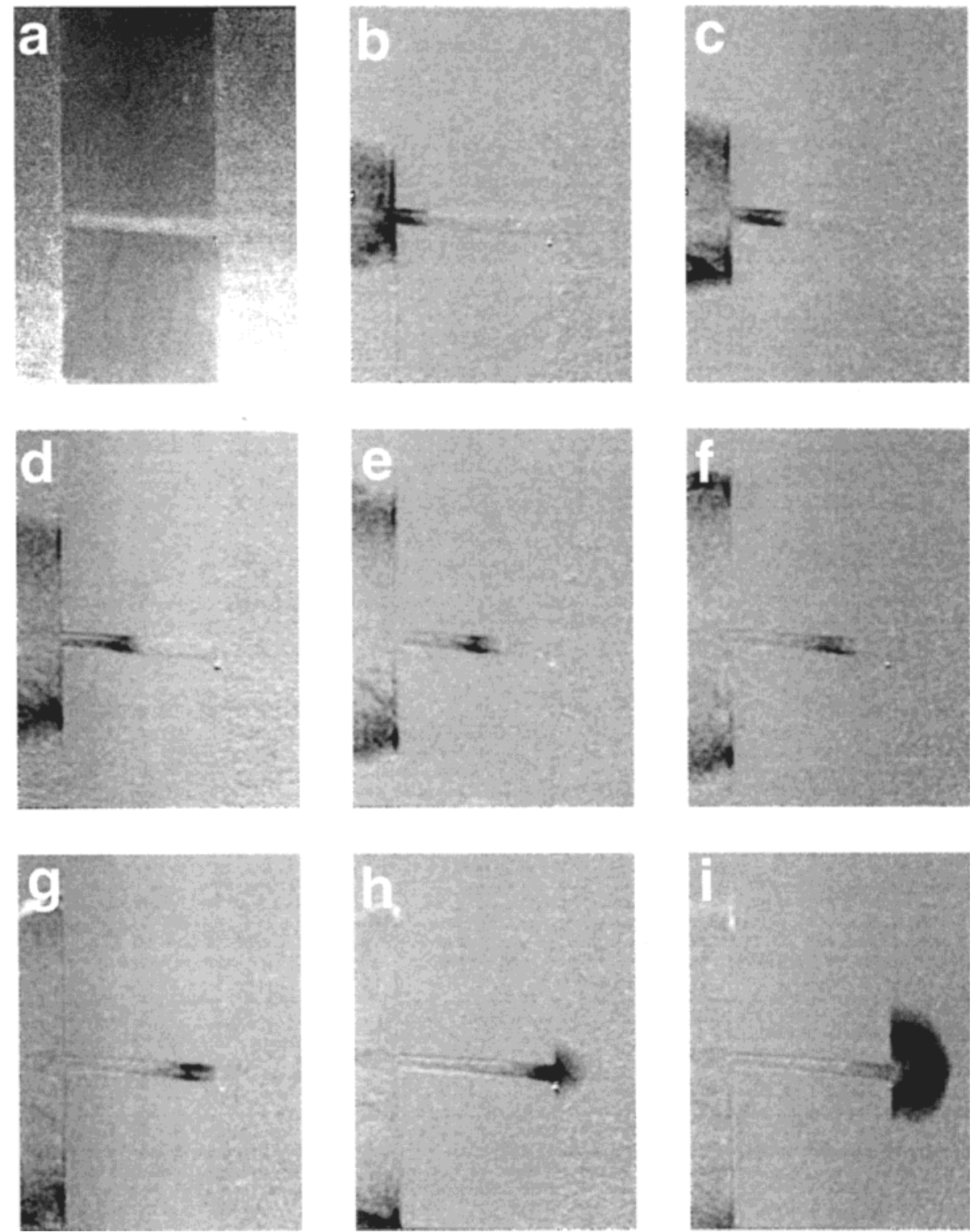

Figure 4. Successful propagation of an electrochemical wave through an unprotected channel. At the exit of the channel, the small dissolution pulse transforms into a semi-circular front. (a) Bright-field image of the obstacle-patterned metal surface; (b-i) sequence of image-processed frames in which dark gray levels indicate regions of increasing dissolution rate. Field of view, $3.4 \times 4.9 \mathrm{~cm}^{2}$; time between frames, $0.4 \mathrm{~s}$; [HNO $\left.\mathrm{H}_{3}\right]$ $=11.9 \mathrm{M}$.

numbers of dissolution waves. Another interesting feature is that the waves reduce the surface roughness in the unprotected areas, which explains the aforementioned difference in the optical characteristics. This effect is most noticeable for the first 10 waves, whereas subsequent dissolution events do not succeed in further "polishing" the surface, possibly because of inhomogeneities of the complex steel structure.

Figure 3 shows the change in surface height at the obstacle as obtained from experiments with different numbers of waves. The plot reveals a proportional dependence between these two quantities and yields an average height loss of $850 \pm 50 \mathrm{~nm} /$ wave. On the basis of the density of the low-carbon steel (7.8 $\left.\mathrm{g} / \mathrm{cm}^{3}\right)$, this value translates into an average material consumption rate of $6.7 \mathrm{~g} /\left(\mathrm{m}^{2}\right.$ wave $)$. The corresponding data are shown in the inset of Figure 3. The largest deviation (12\%) from the average material consumption rate is found for the first five waves. This still minor deviation is possibly related to the decrease in surface roughness described in the context of Figure 2 because a microscopically uneven surface is more susceptible to electrodissolution and gives rise to a slightly increased material consumption rate. It should be noted that the electrochemical smoothening (electropolishing) and the electroetching of surfaces are well-known phenomena, which are used for a broad variety of applications. ${ }^{38}$ Nevertheless, neither of these two phenomena have been discussed in the literature for the case of electrochemical wave propagation.

The results presented in Figures 1-3 clearly establish that the insulating film acts as an adequate obstacle, which locally prevents electrodissolution but allows wave propagation even in very close distance to its boundary. Nonetheless, they also reveal a potential problem which could arise from variations in surface height within the immediate vicinity of the boundary. This feature, however, is intrinsic to any electrodissolution process and therefore unavoidable. To minimize complications that could conceivably arise from these surface variations, the following data are obtained from experiments in which each sample is used for less than 10 wave initiations.

Another interesting feature of obstacle-patterned electrodissolution systems is found from experiments on wave propagation through narrow channels. Figure 4 summarizes a 

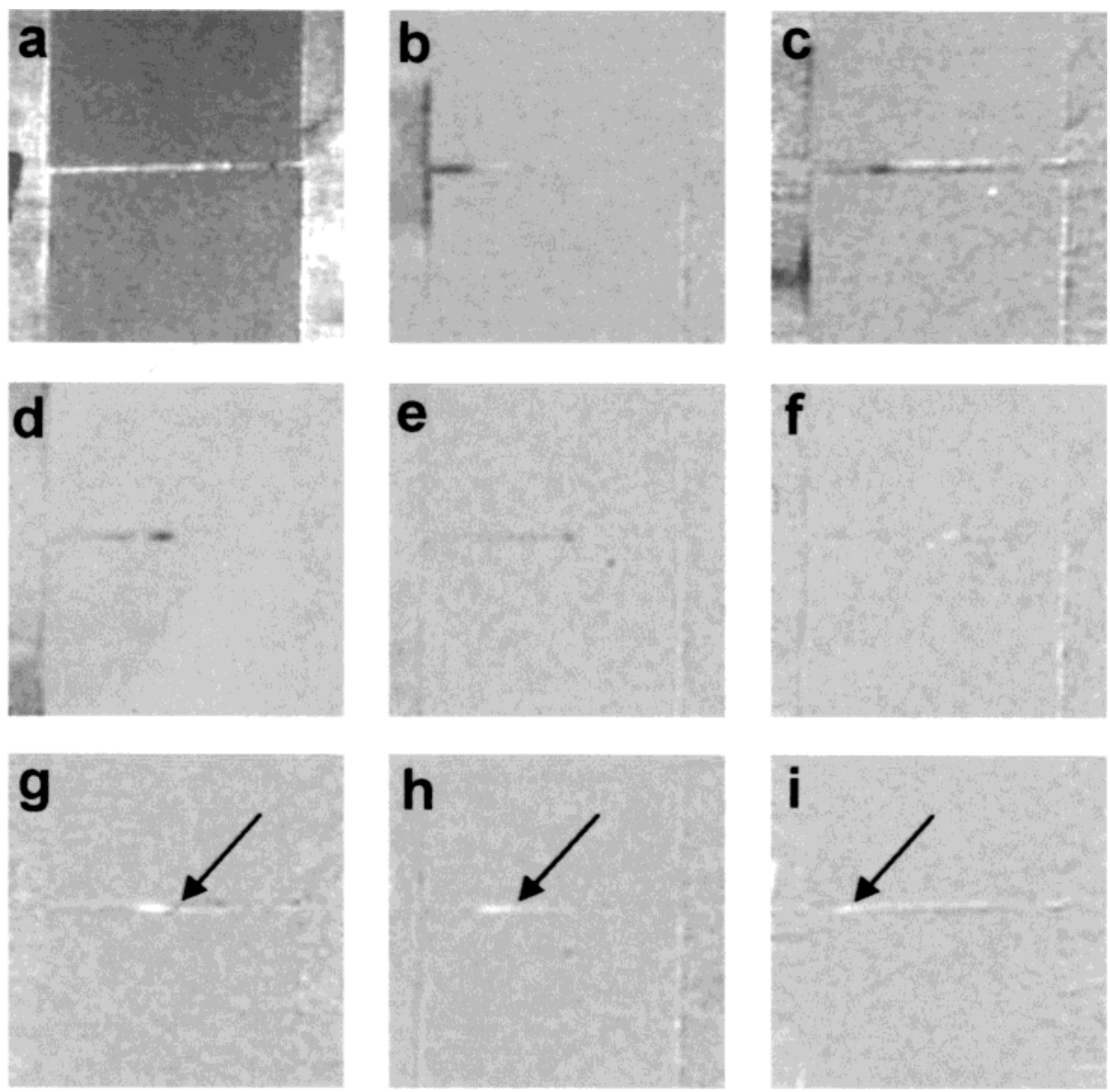

Figure 5. Unsuccessful attempt of an electrochemical wave to propagate through an unprotected channel. (a) Bright-field image of the obstaclepatterned metal surface; $(\mathrm{b}-\mathrm{i})$ sequence of image-processed frames in which dark gray levels indicate regions of increasing dissolution rate. The dissolution front vanishes between frames (e) and (f), and a repassivation pulse (white areas) is formed that travels in reverse direction (see black arrows). Field of view, $2.6 \times 2.3 \mathrm{~cm}^{2}$; time between frames, $0.4 \mathrm{~s}$; $\left[\mathrm{HNO}_{3}\right]=11.9 \mathrm{M}$.

typical experiment in which a wave propagates successfully from one large domain into another by passing through a relatively wide corridor. The shape of the insulating obstacles is shown in Figure 4a. The length and the average width of the passage are 19.0 and $2.0 \mathrm{~mm}$, respectively. Figure $4 \mathrm{~b}-\mathrm{i}$ shows a sequence of eight images in which a solitary wave enters (b) and traverses the channel $(\mathrm{d}-\mathrm{g})$ successfully. Once the confined wave segment has reached the exit (h), it transforms into a semicircular wave (i), which continues to propagate through the unprotected, right domain. The time between consecutive snapshots is $0.4 \mathrm{~s}$. To enhance the clarity of the presented data, subsequent video frames $(\Delta t=0.2 \mathrm{~s})$ were subtracted from each other. This subtraction generates low gray levels in regions in which the image intensity decreases with time. Therefore, dark areas indicate the front position.

The behavior of the wave within the channel depends profoundly on the width of the unprotected corridor. Figure $5 \mathrm{~b}-\mathrm{i}$ shows a sequence of eight consecutive snapshots from an experiment in which the channel width $(0.25 \mathrm{~mm})$ is significantly smaller than that in the example of Figure 4. Like in the earlier example, a bright-field image of the obstacle is given in Figure 5a and the following data $(\mathrm{b}-\mathrm{i})$ are obtained by image subtraction. Under the constraints of this narrow pathway, electrochemical wave propagation fails and the dissolution front does not succeed in exiting into the right domain. Notice that the propagation failure occurs not at the end of the channel but approximately $11.0 \mathrm{~mm}$ away from the entrance, which corresponds to $60 \%$ of the channel's total length. Figure 5 illustrates an another remarkable phenomenon, which we only observed in the case of propagation failure. After the propagation of the dissolution front has stopped, the channel remains in a state of active dissolution. This intermediate state is not well represented by the image-subtracted data because it does not involve any local intensity changes and simply generates a homogeneously gray picture (Figure 5f). In the following, however, a white "echo wave" is observed that travels in reverse direction back toward the entrance of the channel (compare Figure $5 g-i$ ). This phase-wave-like repassivation process appears to be more rapid within the confined geometry of the channel. More importantly, the propagation direction of the repassivation front is highly unexpected because the area around the entrance was activated prior to the area close to the last position of the annihilated front. The detailed dynamics observed in this type of experiment indicate that the obstacles influence the propagation of dissolution waves in a complex fashion. The boundary conditions along the obstacles are clearly not associated with a simple no-flux constraint but are rather reminiscent of sink-type boundaries in the Belousov-Zhabotinsky reaction. ${ }^{29,32}$

To further clarify this important question, we carried out systematic measurements of the wave speed in long, narrow channels. Figure 6 shows the propagation velocity of solitary electrodissolution waves as a function of the channel width for two slightly different concentrations of nitric acid. For a channel width larger than $2.5 \mathrm{~mm}$, the wave speed is unaffected by the presence of the insulating obstacles and equals $10 \mathrm{~mm} / \mathrm{s}$. For narrower channels, however, the velocity decreases with decreasing width down to a value of $4-5 \mathrm{~mm} / \mathrm{s}$. If the channel width is further decreased, propagation failures, similar to the 


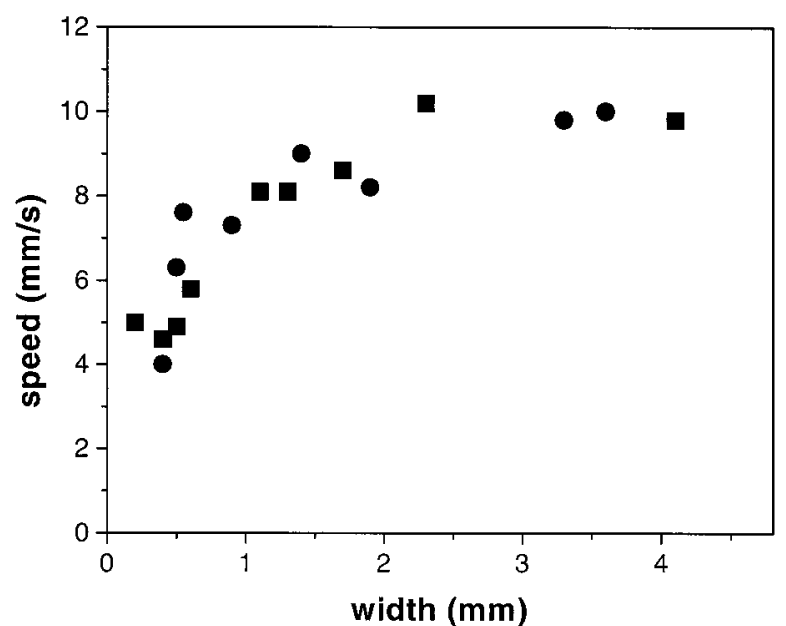

Figure 6. Average speed of electrodissolution waves in long, narrow channels which decreases with decreasing channel width. Solid squares and circles represent data from experiments with $\left[\mathrm{HNO}_{3}\right]=11.2$ and 11.9 M, respectively. Propagation failures are observed for channels narrower than $0.2 \mathrm{~mm}(11.2 \mathrm{M})$ and $0.4 \mathrm{~mm}(11.9 \mathrm{M}$; for details, see text). Length of the channel, $1.9 \mathrm{~cm}$.

one shown in Figure 5, are observed. We could not resolve a significant dependence of the propagation velocity on the concentration of nitric acid. However, our data indicate that the acid concentration affects the critical width below which propagation failures occur. These critical values are found to be 200 and $400 \mu \mathrm{m}$ for $\left[\mathrm{HNO}_{3}\right]=11.2$ and $11.9 \mathrm{M}$, respectively.

The results presented in Figure 6 support our earlier suggestion of a sink-type character of the employed obstacles. We want to emphasize that the dependence of the propagation velocity on the channel width is caused by the dilution of transport fluxes within the dissolution wave. This effect has to be distinguished from propagation failures that occur along convex waves with high local front curvature. The latter phenomenon has been intensively studied for excitable reaction-diffusion media because it yields valuable information on the characteristics of the system including the critical size of circular, super-threshold perturbations. ${ }^{34-36}$ The challenge to identify and characterize the analogy of this critical value for electrodissolution systems is worthwhile because it would specify the critical size of localized defects of the passivation film. The channel geometry of Figures 4 and 5, however, are not appropriate for an estimation of this critical size because the amplitude of the dissolution front is affected by the propagation through the relatively long corridor. We therefore implemented a shorter, gate-like version of the channels, which will be discussed in the following.

Figure 7 illustrates the successful propagation of an electrochemical wave through the narrow opening of such a gate. In this image-processed sequence, the time between subsequent frames is $0.4 \mathrm{~s}$. The first frame (Figure 7a) shows a kidneyshaped zone of repassivation (white area) left behind by a solitary electrodissolution wave which was initiated by scratching the passivated steel surface with a zinc ribbon. Figure $7 \mathrm{~b}$ reveals a small half-circular zone of dissolution (dark area) that is generated as the wave exits from the gap of the obstacle. As shown in Figure 7c-f, this wave continues to expand into the right domain where it eventually vanishes at the edges of the steel plate. In this particular example, the gap has a width of $0.6 \mathrm{~mm}$ and a length of $2 \mathrm{~mm}$. For the given experimental conditions, these parameters are obviously super-critical in the sense that wave propagation prevails despite an intervening period of increased front curvature.

Compared to the case of wave propagation through long channels, the decrease of the wave amplitude within the opening of the gate is greatly reduced. Nevertheless, waves can still vanish at the opening of the gate and, hence, fail to propagate
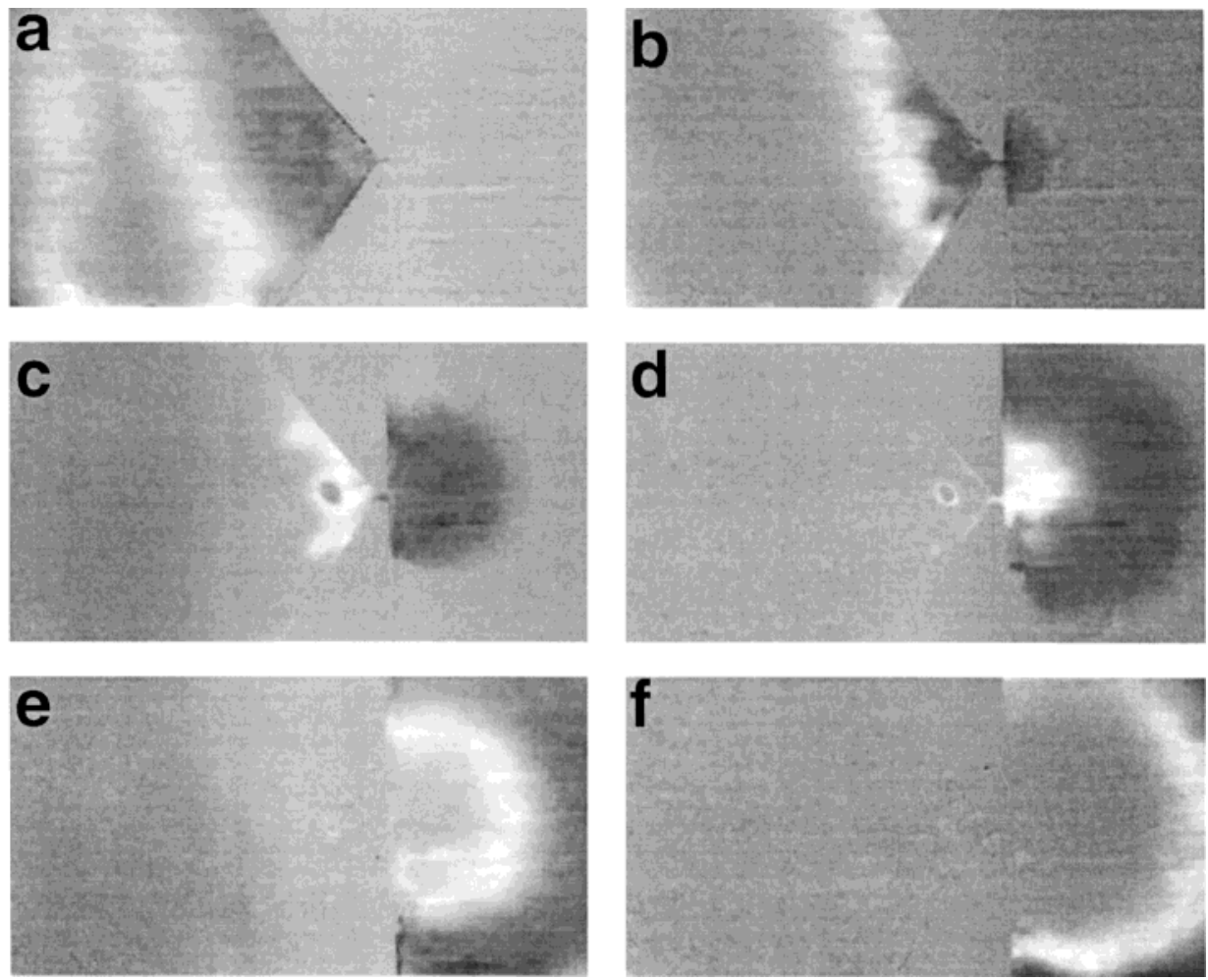

Figure 7. Successful propagation of an electrochemical wave through the unprotected gap of a gate-shaped obstacle. Here, the gap has length of $2 \mathrm{~mm}$ and a width of $0.6 \mathrm{~mm}$. Dark and bright halftones indicate areas of increasing and decreasing dissolution rate, respectively. Field of view, $7.4 \times 3.9 \mathrm{~cm}^{2}$; time between frames, $0.4 \mathrm{~s} ;\left[\mathrm{HNO}_{3}\right]=11.9 \mathrm{M}$ 

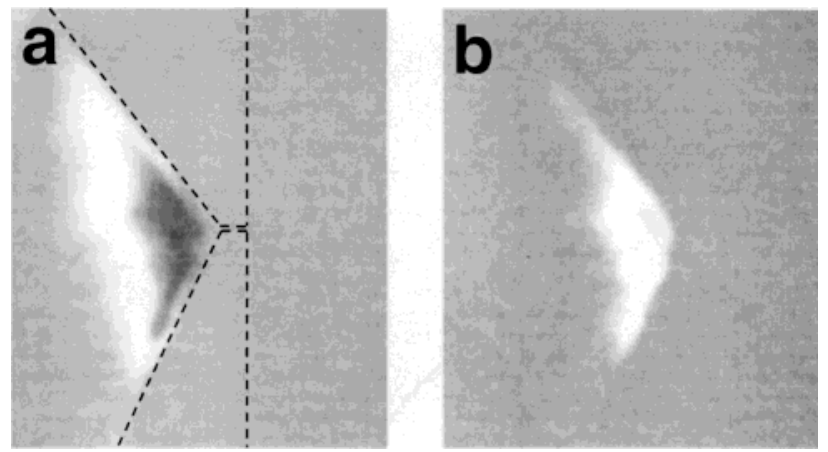

Figure 8. Example for a propagation failure at the narrow opening of a gate-shaped obstacle. The dashed lines in (a) indicate the shape of the insulating obstacle. Field of view, $3.5 \times 4.1 \mathrm{~cm}^{2}$; time between frames, $0.4 \mathrm{~s} ;\left[\mathrm{HNO}_{3}\right]=11.9 \mathrm{M}$; length and width of the unprotected gap, 2.0 and $0.3 \mathrm{~mm}$, respectively.

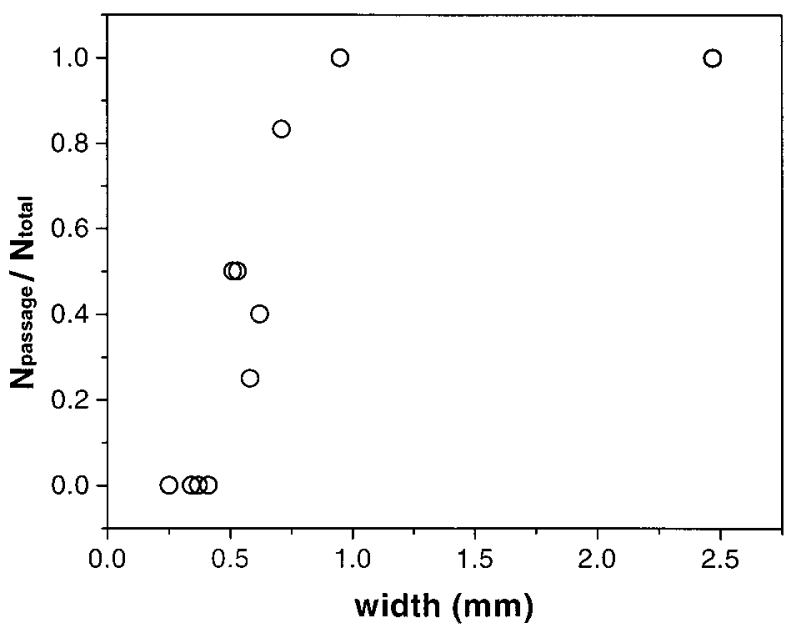

Figure 9. The occurrence of propagation failures strongly depends on the width of the unprotected gap. The abscissa denotes the fraction of the number of experiments in which no propagation failures occurred with respect to the total number of experimental runs, i.e., unsuccessful passage through the obstacle is represented by a ratio of zero. The ordinate denotes the width of the unprotected gap within the gate-shaped obstacles. Length of the gap, $2.0 \mathrm{~mm} ;\left[\mathrm{HNO}_{3}\right]=11.9 \mathrm{M}$.

into the adjacent, unobstructed domain. A typical example for this behavior is shown in Figure 8. Here, the gap width $(0.3$ $\mathrm{mm}$ ) is smaller than that in the experiment of Figure 7. The first frame (Figure 8a) shows a dark region of active dissolution, which approaches the opening of the gate-shaped obstacle from the left. The adjacent white band arises from the trailing zone of repassivation. The subsequent frame (Figure $8 \mathrm{~b}$ ) shows no indications for a significant dissolution of metal in the right domain and the repassivation zone on the left extends to the boundary of the obstacle. Seconds later, the entire surface of the metal is repassivated.

Numerous experiments were carried out to measure the critical gap width for this type of obstacles. These measurements show that waves are reliably blocked by openings with a width smaller than $0.4 \mathrm{~mm}$. On the other hand, waves pass through the obstacle if the gap is larger than $1.0 \mathrm{~mm}$. For gap sizes within the intermediate range of $0.4-1.0 \mathrm{~mm}$, the experimental results are strongly affected by small perturbations. These findings are summarized in Figure 9. The plot shows, for different values of the width, the fraction of experiments in which waves successfully passed through the opening of the obstacle, i.e., the ratios of zero and one correspond to blockage only and passage only, respectively. The presented data allow us to estimate the critical diameter of highly curved, convex dissolution fronts as $(0.65 \pm 0.1) \mathrm{mm}$.

\section{Discussion}

Only sparse data exist regarding the influence of boundary conditions on spatially inhomogeneous processes on electrode surfaces, and the few studies on this intriguing topic are usually limited to the case of long-range (global) coupling. ${ }^{39,40}$ Our results therefore close an important gap, in particular, since they are obtained for wave propagation in a system with local (i.e., reaction-diffusion-like) coupling. Moreover, this study provides the first quantitative data for the wave-dependent material consumption in an open-circuit ${ }^{41}$ system and reveals the existence of two types of propagation failures in electrochemical systems. The first one occurs within the confined geometry of narrow channels and involves an unusual "echo-like" repassivation behavior. The second type of propagation failure is observed at the exit of much shorter, gate-shaped obstacles. On the basis of the presented measurements of wave-induced material consumption, we are confident that our experimental procedure excludes artifacts that could conceivably arise from changes in the surface height around the boundary of the obstacles.

The occurrence of propagation failures is closely related to the characteristic features of the spatial coupling in electrodissolution systems. It is widely accepted that the interfacial potential and the concentration of accompanying electroactive species act as the autocatalytic activator and inhibitor variables, respectively. ${ }^{42-46}$ The wave front is a spatial inhomogeneity in these variables, which induces migration and diffusion fluxes. The time scales associated with these fluxes differ significantly and give rise to a fast transport of the activator and a slow transport of the inhibitor. Accordingly, the wave propagation is dominated by fast electromigration but also influenced by slow diffusion. This caricature of electrochemical wave propagation allows us to qualitatively discuss the nature of the obstacle-wave interaction.

The observation of propagation failures within a narrow channel (Figure 5) implies that the edges of the employed obstacles are not Neumann (no-flux) boundaries because the dynamics of a front oriented perpendicular to a Neumann boundary does not differ from that of an infinitely long front. ${ }^{47}$ Hence, the insulating obstacles influence the propagation of the dissolution front in a more complex fashion by modifying the potential distribution and/or changing the diffusive flux. These factors lead to a decrease in the wave amplitude, which decelerates the electrochemical wave (Figure 6). If the amplitude drops below a critical potential, the wave propagation ceases and a propagation failure occurs. The subsequent formation of an "echo-like" repassivation zone (Figure 5g-i), which moves back toward the entrance of the channel, seems to be closely related to the continuous decrease of amplitude within the channel. One explanation is that the onset of the repassivation process is influenced by the amplitude of the electrochemical wave in the sense that low amplitudes induce premature repassivation. Nonetheless, the origin of the unusual repassivation dynamics in narrow channels could also involve slow diffusive transport between the channel and the adjacent obstacles.

The second type of propagation failure occurs at the narrow opening of relatively short obstacles (Figure 8). These gateshaped barriers were investigated to minimize the decrease in wave amplitude that occurs as the wave passes through the unprotected gap. This type of obstacle also influences the 
electrochemical features of the system, but the undesired perturbations are expected to be of minor relevance. We suggest that in this case the propagation failure occurs immediately after the dissolution front exits from the gap. During this stage, the wave attempts to form a highly curved, semicircular front in which the fluxes in forward direction are significantly diminished. This "defocussing" of electromigration appears to be the most probable candidate for the origin of the observed propagation failures (Figures 8 and 9).

The critical width below which waves fail to pass through the gate-shaped obstacle is approximately $0.65 \mathrm{~mm}$. In this context, it should be noted that comparable experiments under potentiostatic control could increase the reproducibility and, hence, the accuracy of the measurement (see Figure 9). Although the above value is obtained for one particular set of experimental parameters (i.e., steel quality, concentration of nitric acid, etc.), it seems reasonable to assume that its magnitude is representative for a broader spectrum of conditions. Furthermore, we suggest that this critical value could have a significantly more general relevance. The underlying problem can be formulated as a simple question: "What is the minimal size of a defect that can activate a large portion of a passivated metal surface?" or alternatively "What is the maximal size of a defect that can undergo self-healing and reconstruct its passive state without affecting other areas of the metal surface?" For the classic ironnitric acid system, an approximate answer to this intriguing question is provided by the critical size of half-circular waves generated at the gate-shaped obstacles. However, this exploratory study is only one of the first steps toward a more thorough understanding of the nucleation and propagation of electrodissolution waves as well as of their dynamics on surfaces with nontraditional constraints.

Acknowledgment. This work was supported by The Florida State University.

\section{References and Notes}

(1) Scott, S. K. Chemical Chaos; Oxford University Press: Oxford, U.K., 1991.

(2) Kapral, R., Showalter, K., Eds.; Chemical Waves and Patterns; Kluwer: Dordrecht, The Netherlands, 1995. 1173.

(3) Merzhanov, A. G.; Rumanov, E. N. Rev. Mod. Phys. 1999, 71,

(4) Winfree, A. T. Science 1972, 175, 634. 5895 .

(5) Manz, N.; Müller, S. C.; Steinbock, O. J. Phys. Chem. 2000, A104,

(6) Graham, M. D.; Kevrekidis, I. G.; Asakura, K.; Lauterbach, J.; Krischer, K.; Rotermund, H. H.; Ertl, G. Science 1994, 264, 80.

(7) Ertl, G. Adv. Catal. 2000, 45, 1.

(8) Hudson, J. L.; Tsotsis, T. T. Chem. Eng. Sci. 1994, 49, 1493.

(9) Krischer, K. In Modern Aspects of Electrochemistry; Conway, B. E., Bockris, J. O., White, R., Eds.; Kluwer/Plenum: New York, 1999; Vol. $32, \mathrm{p} 1$.
(10) Krischer, K.; Mazouz, N.; Grauel, P. Angew. Chem. 2001, 40, 850. (11) Lev, O.; Sheintuch, M.; Pismen, L. M.; Yarnitzky, C. Nature 1988, $336,488$.

(12) Green, B. J.; Hudson, J. L. Phys. Rev. 2001, E63, 026214

(13) Strasser, P.; Christoph, J.; Lin, W. F.; Hudson, J. L. J. Phys. Chem. 2000, A104, 1854.

(14) Otterstedt, R. D.; Jaeger, N. I.; Plath, P. J.; Hudson, J. L. Chem. Eng. Sci. 1999, 54, 1221.

(15) Flätgen, G.; Krischer K. Phys. Rev. 1995, E51, 3997.

(16) Christoph, J.; Strasser, P.; Eiswirth, M.; Ertl, G. Science 1999, 284, 291

(17) Mazouz, N.; Flätgen, G.; Krischer, K. Phys. Rev. 1997, E55, 2260.

(18) Lillie, R. S. Science 1918, 48, 51.

(19) Bonhoeffer, K. F. J. Gen. Physiol. 1948, 32, 69.

(20) Franck, U. F. Z. Elektrochem. 1958, 62, 649.

(21) Suzuki, R. Adv. Biophys. 1976, 9, 115.

(22) Kaesche, H. Die Korrosion der Metalle; Springer: Berlin, 1990

(23) Kreysa, G., Eckermann, R., Eds.; Dechema Corrosion Handbook; DECHEMA/VCH: Weinheim, 1991; Vol. 10.

(24) Agladze, K.; Thouvenel-Romans, S.; Steinbock, O. Phys. Chem. Chem. Phys. 2001, 3, 1326.

(25) Otterstedt, R. D.; Plath, P. J.; Jaeger, N. I.; Hudson, J. L. Phys. Rev. 1996, E54, 3744.

(26) Otterstedt, R. D.; Plath, P. J.; Jaeger, N. I.; Hudson, J. L. J. Chem. Soc., Faraday Trans. 1996, 92, 2933.

(27) Agladze, K.; Steinbock, O. J. Phys. Chem. 2000, A104, 9816.

(28) Hartmann, N.; Kevrekidis, Y.; Imbihl, R. J. Chem. Phys. 2000, 112,6795

(29) Steinbock, O.; Kettunen, P.; Showalter, K. Science 1995, 269, 1857.

(30) Agladze, K.; Keener, J. P.; Müller, S. C.; Paniflov, A. Science 1994, 264,1746

(31) Jung, P.; Cornell-Bell, A.; Moss, F.; Kadar, S.; Wang, J.; Showalter, K. Chaos 1998, 8, 567.

(32) Suzuki, K.; Yoshinobu, T.; Iwasaki, H. J. Phys. Chem. 2000, A104, 6602

(33) Suzuki, K.; Yoshinobu, T.; Iwasaki, H. J. Phys. Chem. 2000, A104, 5154.

(34) Tóth, Á.; Gaspar, V.; Showalter, K. J. Phys. Chem. 1994, 98, 522.

(35) Foerster, P.; Müller, S. C.; Hess, B. Proc. Natl. Acad. Sci. U.S.A. 1989, 86,6831 .

(36) Lewis, T. J.; Keener, J. P. SIAM J. Appl. Math. 2000, 61, 293.

(37) An exception is recent studies on arrays of globally coupled electrodes, e.g., Kiss, I. Z.; Wang, W.; Hudson, J. L. J. Phys. Chem. 1999, B103, 11433 .

(38) Lainer, V. I. Modern Electroplating; (translated from Russian) U.S. Department of Commerce: Washington, D.C., 1970.

(39) Wave-initiation at the rim of disk electrodes was first reported in Pigeau, A.; Kirkpatrick, H. B. Corrosion 1969, 25, 209.

(40) Hudson, J. L.; Tabora, J.; Krischer, K.; Kevrekidis, I. G. Phys. Lett. 1993, A179, 355

(41) Horanyi, G.; Inzelt, G.; Szetey, E. Acta Chim. Hung. 1978, 97, 299.

(42) Franck, U. F.; FitzHugh, R. Z. Elektrochem. 1961, 65, 156

(43) Koper, M. T. M.; Sluyters, J. H. J. Electroanal. Chem. 1993, 347,

(44) Koper, M. T. M.; Sluyters, J. H. Electrochim. Acta 1993, 38, 1535.

(45) Flätgen, G.; Krischer, K. J. Chem. Phys. 1995, 103, 5428.

(46) Birzu, A.; Green, B. J.; Otterstedt, R. D.; Jaeger, N. I.; Hudson, J. L. Phys. Chem. Chem. Phys. 2000, 2, 2715.

(47) Formally, a straight, no-flux boundary generates a virtual mirror image of the main system. 